\title{
Calculating the consequences of recovery, a European model for inhabited areas
}

\author{
T.W. Charnock ${ }^{1}$, J.A. Jones ${ }^{1}$, L.N. Singer ${ }^{1}$, K.G. Andersson ${ }^{2}$, \\ J. Roed ${ }^{2}$, S. Thykier-Nielsen ${ }^{2}$, T. Mikkelsen ${ }^{2}$, P. Astrup ${ }^{2}$, J.C. Kaiser ${ }^{3}$, \\ H. Müller ${ }^{3}$, G. Pröhl ${ }^{3}$, W. Raskob ${ }^{4}$, S.C. Hoe $^{5}$, L.H. Jacobsen ${ }^{6}$, \\ L. Schou-Jensen ${ }^{6}$ and F. Gering ${ }^{7}$ \\ ${ }^{1}$ Health Protection Agency, Centre for Radiation, Chemical and Environmental Hazards, \\ OX11 ORQ Chilton, Didcot, Oxon, UK \\ ${ }^{2}$ Risø National Laboratory for Sustainable Energy, Technical University of Denmark, \\ PO Box 49, 4000 Roskilde, Denmark \\ ${ }^{3} \mathrm{Helmholtz-Zentrum} \mathrm{Muenchen,} \mathrm{Ingolstädter} \mathrm{Landstr.} \mathrm{1,} 85764$ Neuherberg, Germany \\ ${ }^{4}$ Forschungszentrum Karlsruhe, IKET, Bau 433, Postfach 3640, 76021 Karlsruhe, Germany \\ ${ }^{5}$ DEMA - Danish Emergency Management Agency, Datavej 16, 3460 Birkerød, Denmark \\ ${ }^{6}$ PDC - Prolog Development Center, H.J. Holst Vej 3C - 5C, 2605 Brøndby, Denmark \\ ${ }^{7}$ Bundesamt für Strahlenschutz - SW 3.2, Ingolstädter Landstr. 1, \\ 85764 Oberschleißheim, Germany
}

\begin{abstract}
The European Model for Inhabited Areas (ERMIN) was developed to allow a user to explore different recovery options following the contamination of an urban environment with radioactive material and to refine an appropriate strategy for the whole region affected. The input data include a description of the environment, initial deposition of radionuclides on to a reference surface and a description of countermeasures. Output information includes the average doses to members of the public from external exposure to gamma and beta radiation from deposited radionuclides and inhalation of resuspended radioactivity, the contamination on urban surfaces, the activity concentration in air from resuspension, the doses to workers undertaking the recovery work, the quantity and activity of waste generated and the cost and work required to implement the countermeasure. ERMIN has been designed to be implemented as a tool that supports the approach of decision-makers and allows the area to be broken down into smaller regions where different conditions prevail and different countermeasure packages are enacted.
\end{abstract}

\section{INTRODUCTION}

The European Model for Inhabited Areas (ERMIN) was developed under EURANOS [1], an integrated project of the EC Sixth Framework Programme. It is largely complete and is being released as version 1. ERMIN is both a model and a software tool. As a model it simulates the behaviour of radionuclides in the inhabited environment and calculates the exposure of the population as well as other relevant endpoints. As a tool it allows a user to explore different recovery options following the contamination of an urban environment with radioactive material and therefore to refine a strategy. It has been designed to be implemented within the RODOS [2] and ARGOS [3] Nuclear Emergency Decision Support Systems (DSS) as well as a standalone application.

The need for a tool with the capabilities of ERMIN was identified during the EC Fifth Framework programme. In particular, work during the EVATECH project [4] indicated that decision makers recognised the need to address recovery issues by sub-dividing the area of concern on the basis of, for example, land use, deposition level and the emergency actions taken; and to explore the combined consequences of applying different packages of countermeasures within those subdivisions. Such a 
flexible approach could not be readily handled by the tools then available and it was clear that the flexibility required could only be achieved when the user was able to interact directly with a dynamic model.

\section{THE ERMIN MODEL}

Figure 1 illustrates the calculation stages of the ERMIN model. The Input data required include the initial deposition of radionuclides on to a reference surface, a description of the urban environment and a description of countermeasures to be applied. ERMIN can accept deposition from an atmospheric dispersion model, surface measurements and the Inhabited Area Measurement Module (IAMM) - a data assimilation module also developed under EURANOS for implementation in RODOS and ARGOS [5]. Countermeasures include decontamination, fixing radioactivity to a surface, shielding and relocation.

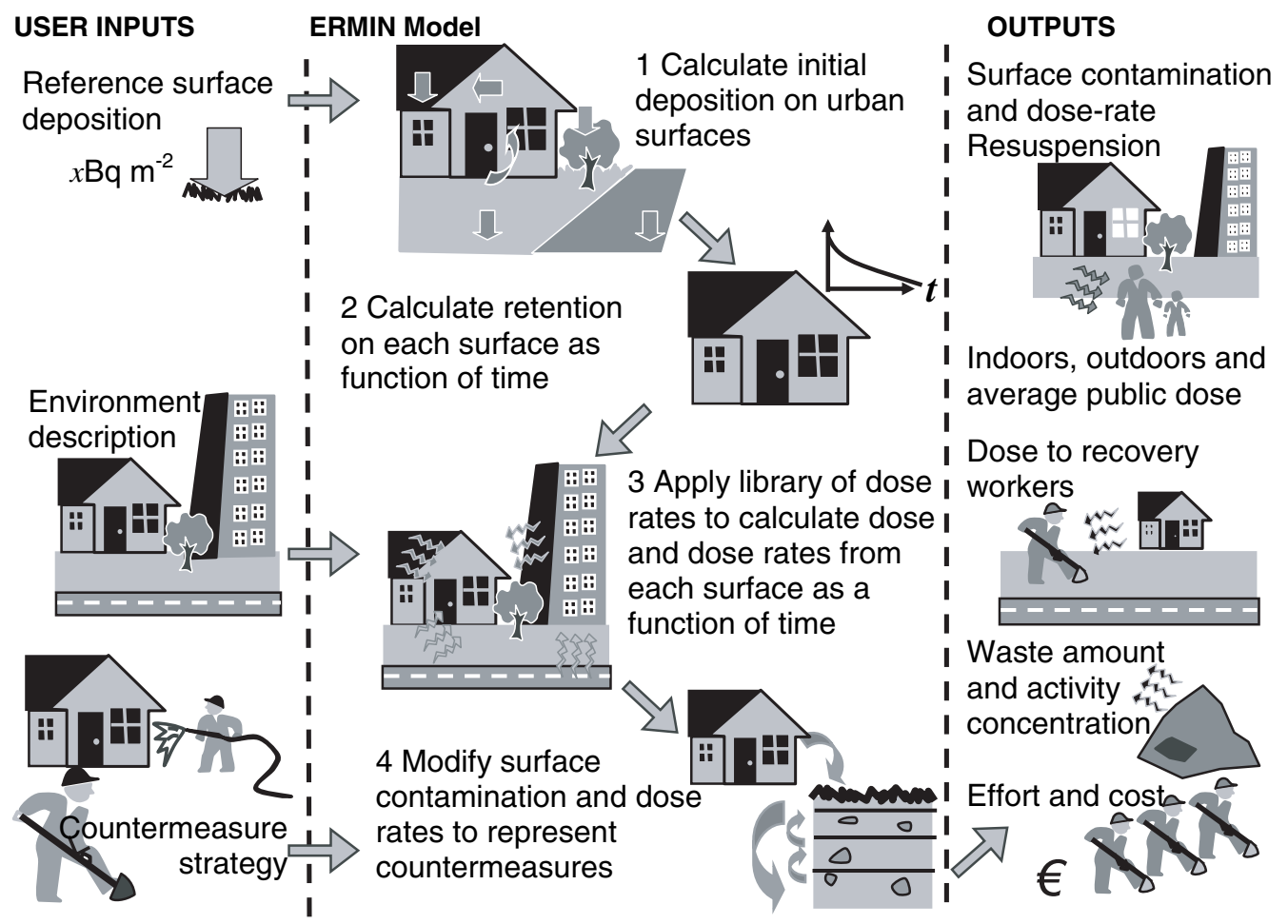

Figure 1. Stages of the ERMIN calculation.

Output information generated by the tool includes the average doses to members of the public from external exposure to gamma and beta radiation from deposited radionuclides and inhalation of resuspended radioactivity, the contamination on urban surfaces, the activity concentration in air from resuspension, the doses to workers undertaking the recovery work, the quantity and activity of waste generated and the cost and work required to implement the countermeasures.

The model calculation can be divided into four stages. First, the model uses weather dependent ratios to distribute the deposition on the reference surface onto all the urban surfaces that it represents, including; trees, roofs, walls, roads and building interiors. Second, empirical functions are used to represent the long-term surface retention and migration in soil is simulated using a convective-dispersive model. Third, a library of dose rates for surfaces in idealised environments is applied to calculate dose 
rates indoors and outdoors. Fourth, countermeasures are represented by the modification of surface contamination and the dose-rates. The ERMIN countermeasure database is based on the EUROPEAN inhabited area handbook also developed under the EURANOS project [6]. In order to represent a spatial varying situation the model is applied repeatedly across a grid covering the area of interest.

\section{IMPLEMENTATION AND OPERATION}

ERMIN was designed to be implemented within both the RODOS and ARGOS Nuclear Emergency DSS and also as a standalone application. Each implementation uses the same underlying executable and data library components, but the tool, as presented to the user, can be very different depending on the needs of the users of a particular system. However the basic principles of operation are the same in that the user defines regions. Regions may represent areas of different urban environments, different deposition, different emergency countermeasures and different packages of recovery options to be evaluated. Within the RODOS and ARGOS DSS the regions are directly drawn onto a map. Figures 2 and 3 show a typical user interaction with the RODOS ERMIN user interface.

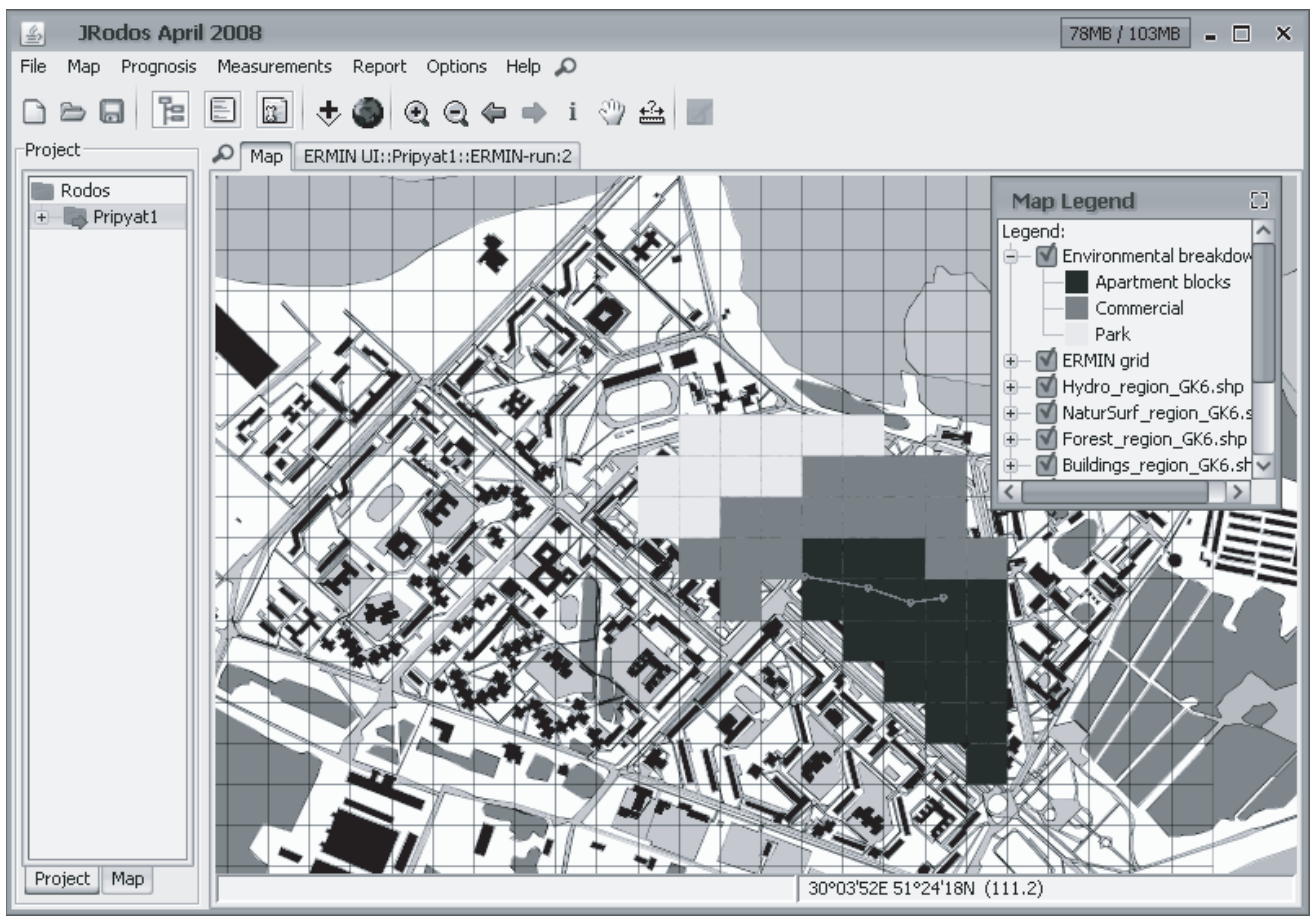

Figure 2. A typical screen shot of the RODOS ERMIN user interface. Here the user delineates three environment regions within the Pripyat settlement, representing areas of apartment blocks, a commercial area and parkland respectively.

The output in RODOS and ARGOS can be delivered as a grid overlaid onto a background map for visualisation, see Figure 4, or summarised over a region. When using ERMIN, the user may make the input description as complicated or as simple as necessary with regard to their immediate needs. For example, for the purposes of response in the early recovery phase, where a broad projection of doses without countermeasures or the evaluation of a few simple countermeasure options is required, it may be appropriate to simply define a town using a single default environment, and to assume that a single countermeasure such as road sweeping is applied everywhere. In the longer term, it may be appropriate 


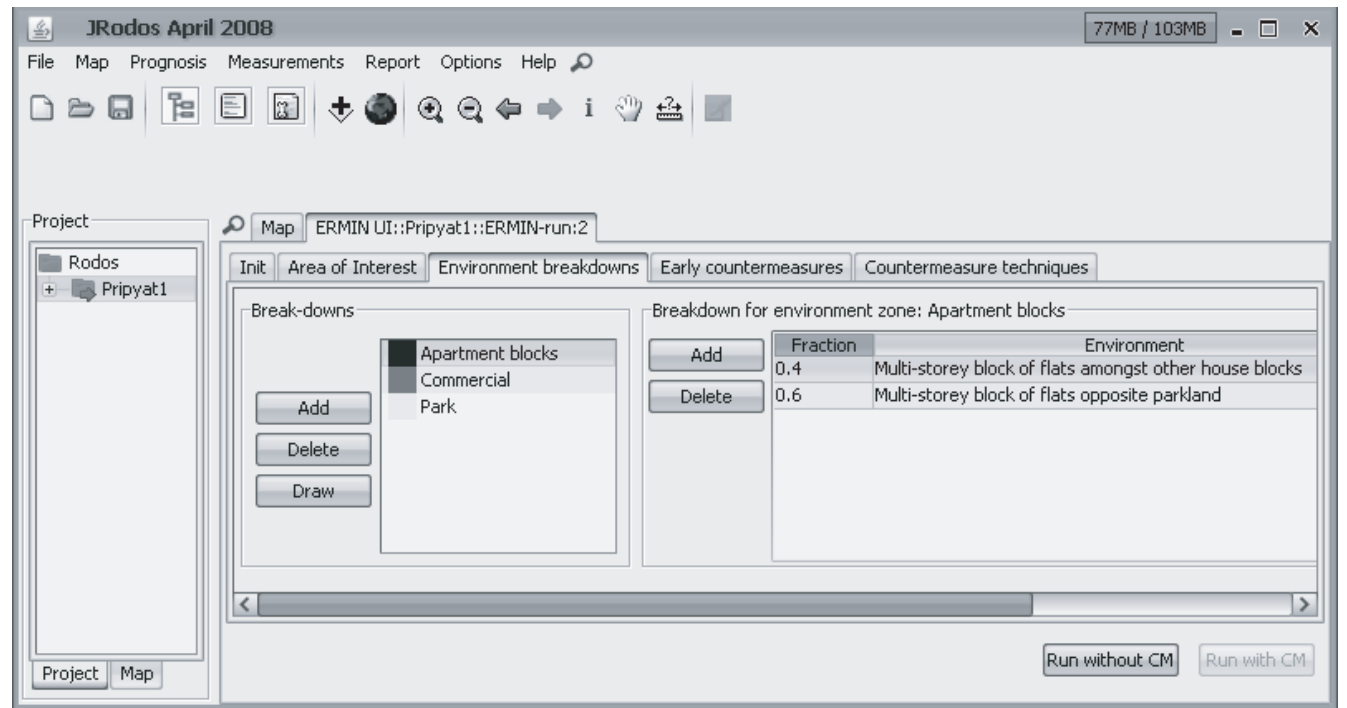

Figure 3. A typical screen shot of the RODOS ERMIN user interface. Here the user specifies that the region "apartment blocks" is represented by a mix of two library environments comprising multi-storey buildings.
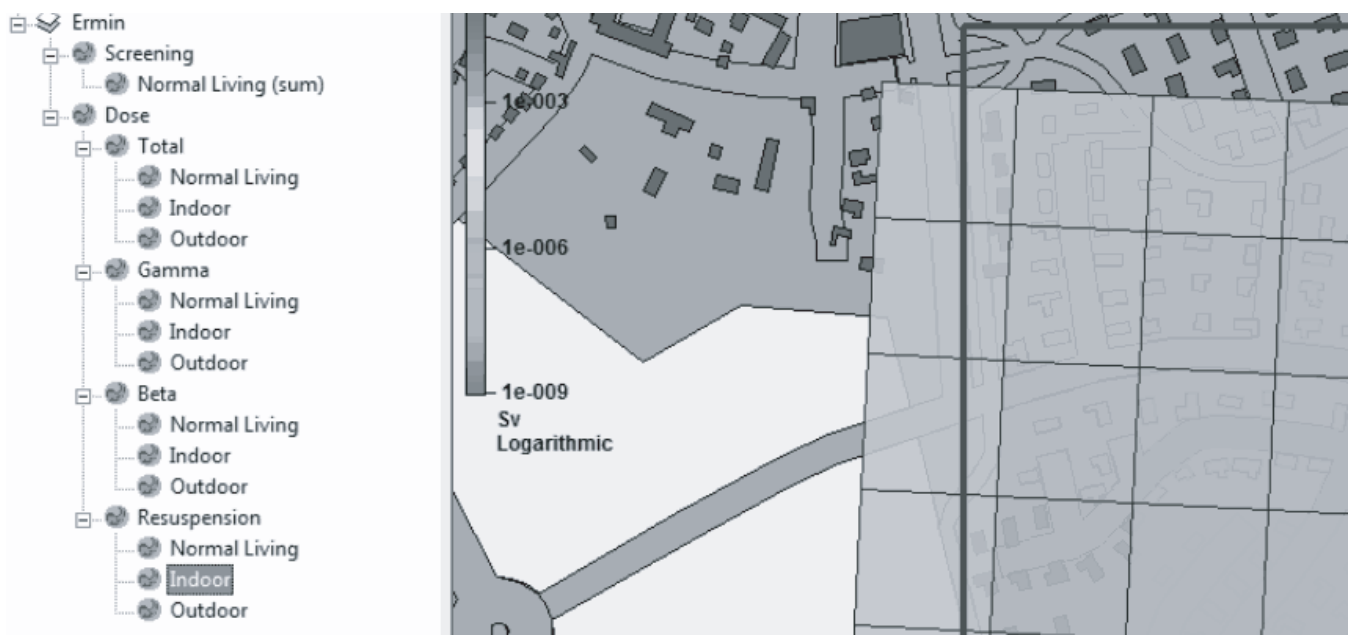

Figure 4. A screen shot from the ARGOS Emergency DSS, showing the visualisation of ERMIN results against background map of an urban area.

to define different environments within different parts of the town and to identify the regions in which countermeasure packages are applied.

\section{TESTING AND COMPARISON}

As part of the model evaluation process, ERMIN was compared with EXPURT [7] using a scenario developed by the Urban Remediation Working Group of the IAEA EMRAS (Environmental Models for Radiation Safety) Programme [8] based on the settlement of Pripyat that was evacuated after the Chernobyl accident. ERMIN produced results largely consistent EXPURT as in Figure 5. 


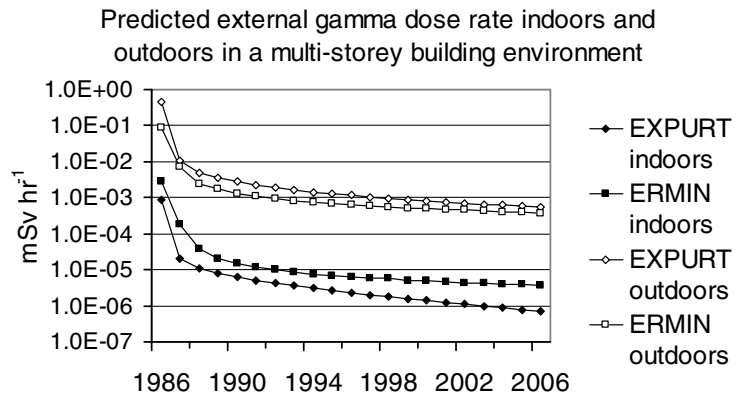

Figure 5. External gamma doses rates in an indoors and outdoor location predicted by ERMIN and EXPURT.

Generally predicted dose rates fall away slightly more rapidly in EXPURT than in ERMIN. This result is not surprising as the ERMIN project included a thorough review [9] of the latest surface retention data and many of the retention parameters were revised to indicate greater retention than the parameters used by EXPURT. EXPURT also tends to have a higher initial dose rate, particularly, outdoors or in less shielded interiors. This is due in part to a larger initial deposition to trees. More recent data has allowed the parameters for initial deposition to trees to be revised downwards for the ERMIN development. Figure 6 compares the contributions of different surfaces to total dose rate and the difference in contributions from trees is apparent.

Fraction of indoor dose rate

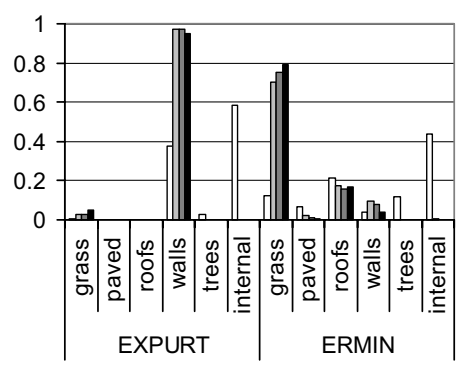

Fraction of outdoor dose rate

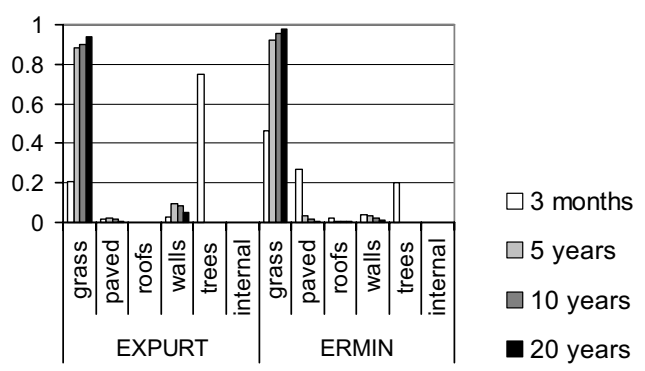

Figure 6. Contributions of surfaces to indoor and outdoor locations predicted by ERMIN and EXPURT. The results are for EXPURT and ERMIN environments that represent multi-storey buildings with high shielding.

Figure 6 shows up another obvious difference between EXPURT and ERMIN in the relative contribution of grass surfaces to indoor dose rate. This difference is due to the configuration of the idealised environments. EXPURT has only one multi-storey building environment that, therefore, has to be used for the Pripyat scenario although it has a building density that is rather too high; hence there is a high contribution from exterior walls. The ERMIN database has two distinct multi-storey environments one similar to the EXPURT environment and one that represents a multi-storey building next to an open green area and which is much more suitable to the Pripyat conditions.

\section{CONCLUSIONS}

The integration of ERMIN with RODOS and ARGOS is an ongoing process, as is the evaluation of the ERMIN model itself. An important part of the process is gathering opinions from the users of the system to ensure that the user interface supports the way the user needs to work and that the outputs are directed towards the decisions that need to be taken. 
The ERMIN v1 database has been designed to be extendable and in future versions of ERMIN it could be enhanced to included additional urban environments as well as retention and deposition parameters suitable for different types of scenarios such as explosions or fires.

\section{Acknowledgments}

Work on the ERMIN software received partial financial support from the European Commission Sixth Framework Programme (Nuclear Fission/Radiation Protection) under the EURANOS integrated project: European approach to nuclear and radiological emergency management and rehabilitation strategies (Contract No: FI6R-CT-2004508843).

\section{References}

[1] Raskob W., Gering F., Lochard J., Nisbet A.F., Starostova V. and Tomic B., Objectives and achievements in the first three years of the EURANOS project: European Approach to Nuclear and Radiological Emergency Management and Rehabilitation Strategies (These proceedings, 2008).

[2] Ehrhardt J. (Ed.), Migration of RODOS to Practical Applicability (Forschingszentrum Karlsruhe in der Helmholtz-Gemeinschaft, Karlesruhe, Germany, FZKA 7015, 2004).

[3] Whitepaper ARGOS CBRN Decision support for emergency management (Prolog development centre, Denmark, available at http://www.pdc.dk/argos/ARGOS_whitepaper.pdf, 2007).

[4] Brown J., Roberts G., Carter E., French S. and Niculae C., Summary report of the UK workshops held to explore decision analysis of clean-up actions in inhabited areas after an accidental release of radionuclides (EVATECH(WP4)-TN(04)-02, 2004).

[5] Kaiser, J.C. and Pröhl G., Kerntechnik 72/4 (2007) 218-221.

[6] Brown, J., Mortimer K., Andersson K., Duranova T., Mrskova A., Hänninen R., Ikäheimonen T., Kirchner G., Bertsch V., Gallay F., Reales N., Hammond D. and Kwakman P., Generic handbook for assisting in the management of contaminated inhabited areas in Europe following a radiological emergency, parts I-VI (EURANOS(CAT1)-TN(07)-02, 2007).

[7] Jones, J.A., Singer L.N. and Brown J., J. Environ. Radioactiv. 85 (2006) 314-329.

[8] IAEA., Environmental Modelling of Remediation of Urban Contaminated Areas, Environmental Modelling for Radiation Safety Programme (EMRAS), (International Atomic Energy Agency. Vienna. to be published).

[9] Jones, J.A., Charnock T.W., Singer L., Roed J., Andersson K., Thykier-Nielsen S., Mikkelsen T., Astrup P., Kaiser J.C., Müller H., Pröhl G., Raskob W., Hoe S., Jacobsen L.H., Schou Jensen L. and Gering F., Description of the Modelling of Transfer and Dose Calculations within ERMIN v1.0 and associated data libraries (EURANOS(CAT2)-TN(05)-04, 2007). 